\title{
Standards of care and clinical predictors in patients hospitalised for a COPD exacerbation - The Italian SOS (Stratification Observational Study)
}

\author{
M. Lusuardi1, F. Blasi2, C. Terzano3, C. Cricelli4, N. Crispino5, L. Comarella6, \\ F. De Benedetto7, C.M. Sanguinetti8, L. Allegra2, C.F. Donner9
}

ABSTRACT: Standards of care and clinical predictors in patients hospitalised for a COPD exacerbation - The Italian SOS (Stratification Observational Study). M. Lusuardi, F. Blasi, C. Terzano, C. Cricelli, N. Crispino, L. Comarella, F. De Benedetto, C.M. Sanguinetti, L. Allegra, C.F. Donner.

Background and aims. Hospitalisations for chronic obstructive pulmonary disease (COPD) exacerbations are major events in the natural history of the disease in terms of survival, quality of life and risk of further episodes of exacerbation. The aims of study were to evaluate:

1. adherence to recommended standards of care; and

2. clinical factors influencing major outcomes during hospitalisation for an episode of COPD exacerbation and within a 6-month follow-up.

Methods. An observational, prospective study was conducted in 68 centres. Assessment of standards of care included diagnostic procedures (such as pulmonary function tests and microbiology) and management options (such as drug therapies, vaccinations and rehabilitation). Outcome measures relevant to the hospitalisation were: survival, need for mechanical ventilation, and length of stay (LOS). Outcomes at 6-months were: survival, exacerbations and hospitalisations for an exacerbation. Multi- variate logistic regression was applied to evaluate the relation between clinical factors and outcomes.

Results. 931 patients were enrolled. Only 556 patients $(59.7 \%)$ were diagnosed COPD and stratified for severity with the support of spirometry $\left(\mathrm{FEV}_{1} / \mathrm{VC} \leq 0.7\right)$ and were considered for outcome analysis. Among treatments, pulmonary rehabilitation and anti-smoking counselling were applied infrequently (14.5 and $8.1 \%$ of patients, respectively). Within six months 63 COPD patients $(17.7 \%)$ had at least one episode of exacerbation prompting a further hospitalisation and 19 died $(5.3 \%)$. Predictor of mortality was the co-morbidity Charlson index (odds ratio, OR 10.3, $p=0.03$ CI:1.25-84.96). A further hospitalisation was predicted by hospitalisation for an exacerbation in the previous 12 months (OR 3.59, $p=0.003$ CI:1.54-8.39).

Conclusions. Standards of care were far lower than recommended, in particular $40 \%$ of patients were labelled as COPD without spirometry. COPD patients with a second hospitalisation in $\mathbf{1 2}$ months for an exacerbation had about 3 times the risk of suffering a new episode and hospitalisation in the following six months.

Monaldi Arch Chest Dis 2009; 71: 4, 153-160.

Keywords: Chronic obstructive pulmonary disease, Exacerbation, Hospitalisation, Guidelines, Standards of care.

${ }^{I}$ Unit of Pulmonary Rehabilitation, San Sebastiano Hospital, Local Health Authority (AUSL) of Reggio Emilia, Correggio, I

2 Institute of Respiratory Diseases, University of Milano, "Maggiore Policlinico" Hospital, Mangiagalli and Regina Elena, Milano,

3 "La Sapienza" University, 1st School of Medicine, Roma,

4 Italian Society of General Medicine (SIMG), Firenze,

5 Pfizer Italia, Roma,

6 Contract Research Organisation Statistics and Data Management (CROS NT), Verona,

7 Department of Pneumology - "Santissima Annunziata" Hospital - Local Health Authority of Chieti,

8 Department of Pneumology, San Filippo Neri Hospital, Roma,

9 Mondo Medico, Multidisciplinary and Rehabilitation Outpatient Clinic, Borgomanero (Novara), Italy.

Correspondence: Mirco Lusuardi, Pulmonary Rehabilitation, S. Sebastiano Hospital, Reggio Emilia AUSL, Via Mandriolo 11, I-42015Correggio (RE), Italy; e-mail: lusuardimi@ausl.re.it

\section{Introduction}

Hospitalisations for chronic obstructive pulmonary disease (COPD) exacerbations are major events in the natural history of the disease in terms of survival, quality of life and risk of further episodes of exacerbation [1-8]. From a societal point of view they represent the main cost in relation to health care resources due to COPD [9], and account for about $10 \%$ of all hospitalisations [10]. The ICE (Italian Costs for Exacerbation in COPD) study confirmed that COPD is a disease with high costs: even at a conservative estimate COPD is responsible for about $6 \%$ of the total health care ex- 
penditure, the largest share of costs being for hospitalisation [11].

Different studies have focused on factors influencing the risk and the outcomes of an exacerbation, in an attempt to plan strategies for minimising the individual and socio-economic impact of COPD exacerbations. Several clinical factors associated with an exacerbation have been reported as predictors of major outcome measures (in particular, survival and hospitalisation): they include lower $\mathrm{FEV}_{1}$, hypoxaemia, lower albumin, lower Body Mass Index (BMI), longer disease duration or shorter time since the first hospitalisation [1-8]. Multidimensional grading systems, such as the BODE (BMI, Obstruction, Dyspnea, Exercise), seem to offer a better prediction of clinical outcomes than individual measures $[12,13]$.

Hospitalisation per se is a major predictor of poor survival, of re-exacerbation risk and re-admission to hospital [7]. On the other hand, in large hospitals with more respiratory consultants and better quality organised care, mortality and length of stay are lower $[14,15]$.

Among treatment factors, under-prescription of Long Term Oxygen Therapy (LTOT) or rehabilitation are both risk factors for a worse outcome [4]. Different pharmacologic trials have suggested better outcomes in patients on regular treatment with inhaled corticosteroids [16] or tiotropium [17]. Last but not least, a reduced hospitalisation rate for COPD exacerbation seems largely influenced by organisational factors, such as integrated/planned care supported by information technology and involving different levels of the health care system [18].

Since medical interventions and organisational factors may influence the risk and the outcomes of a COPD exacerbation, correct knowledge of the actual standards of care and of the main risk factors in each population and health system is mandatory.

The primary aim of the Italian "SOS (Stratification Observational Study) on COPD" was to evaluate the standards of care and clinical factors influencing major outcome measures such as survival, length of stay (LOS), and intensive care unit (ICU) admission, during hospitalisation for an episode of COPD exacerbation in a cohort of patients representative of the Italian COPD population.

The secondary aim was to estimate the risk of a new episode of exacerbation, hospital admission or death during a 6-month follow up.

\section{Materials and methods}

\section{Study Design and Subjects}

An observational, prospective study involving 68 pneumology centres evenly distributed throughout Italy was conducted. Enrolment of patients commenced in October 2004 and lasted for 4 months. The enrolment period had a follow-up interval of 6 months; visits took place after 1 and 6 months at the centre and a phone call was performed after 3 months from hospital discharge.
The first 20 consecutive patients of both sexes, aged $\geq 45$ years who were admitted to each centre for a COPD exacerbation having had a chest X-ray negative for pneumonia, tuberculosis or pulmonary cancer, were enrolled. A COPD exacerbation was defined according to GOLD guidelines, i.e. increased breathlessness, possibly accompanied by wheezing and chest tightness, increased cough and sputum, change of the colour and/or texture of the sputum, and fever [16].

To evaluate actual practice versus standards of care as derived from GOLD guideline recommendations [16], the study did not foresee any definite protocol of patient management, i.e. each centre agreed to perform all diagnostic and treatment procedures as per their usual practice consolidated in time.

In relation to ethical considerations, the study was approved by each local review board. The written consent of each patient was formally requested and obtained regarding the use of personal data for research.

\section{Data collection}

\section{AT HOSPITAL ADMISSION}

Demographic and case-history data were collected for all patients including age, sex, socioeconomic status, risk factors for COPD (in particular smoking and occupational history) and COPD history with particular regard to hospitalisations in the previous 3 months, use of medications for COPD, use of antibiotics and non-pharmacological therapies. Comorbidity was measured for all patients with the Charlson index [19].

Physical examination, spirometric, biochemical and microbiological examinations upon admission were also recorded. Investigators were recommended to record pulmonary function tests preferably obtained in a stable phase of the disease in the 12 months before or 6 months following the index hospitalisation. For a correct COPD diagnosis and severity stratification a $\mathrm{FEV}_{1} / \mathrm{VC} \leq 0.7$ post-bronchodilator was required.

Outcome measures relevant to the hospital stay were: mortality, need for mechanical ventilation, and length of hospital stay (LOS).

\section{AT FOLLOW-UP}

During follow-up visits (at 1 and 6 months) data on COPD exacerbations and hospitalisations related or not to COPD were collected.

During the telephone interview at 3 months from discharge, data on COPD exacerbations and hospitalisations related or not to COPD and deaths were collected.

Outcome measures relevant to the follow up were survival, new episodes of exacerbation and new hospitalisations for an exacerbation.

\section{Statistical Analysis}

Analyses were carried out using SAS ${ }^{\circledR}$ System software (8.2, TS level $02 \mathrm{Mo})$. 
For continuous variables, mean and standard deviation or median with inter-quartile range were calculated as necessary. For dichotomic variables, absolute frequencies and percentages were calculated. Association between each outcome (mortality, need of mechanical ventilation, re-exacerbation, re-admission for exacerbation) and demographic and clinical variables was assessed using multivariate logistic regression models. Factors found to be significant on bivariate logistic regression or judged to be clinically relevant were included in the multivariate models and selected using backward stepwise procedure $(P<0.05$ to enter into the model, $\mathrm{P}<0.10$ to remain). The risk of developing the event in the presence of a particular factor was evaluated in terms of odds ratio (OR), calculated with the corresponding $95 \%$ confidence interval (CI). Results were considered statistically significant at $P<0.05$.

\section{Results}

A total of 931 patients were enrolled at hospital admission for an episode of COPD exacerbation, most of them coming directly from home $(92.5 \%)$, while $5.9 \%$ of patients came from non pneumological hospital units, and $1.2 \%$ from nursing homes or long term health care facilities; 667 were males $(71.6 \%)$, the mean age was 72.2 years ( $\pm 8.7 \mathrm{SD}$, standard deviation). Active smoking was still present in 149 patients $(16.0 \%)$, the majority having ceased at least 6 months before (565, $60.7 \%)$; mean pack-years were $66.3( \pm 35.2)$. About one out of five patients $(200,21.5 \%)$ were stated to have never smoked, while in $17(1.8 \%)$ cases data on smoking habit was not obtained.

Exposure to passive smoking and to professional pollutants was present in $240(25.8 \%)$ and $142(15.3 \%)$ patients respectively, but only 71 among the never-smokers had been exposed to either passive smoking and/or occupational pollutants; therefore in 130 patients $(13.9 \%)$ no exposure to usual exogenous risk factors was demonstrable.

In about $2 / 3$ of the case series (637 patients, $68.4 \%$ ) the diagnosis of COPD was already known, hence 294 patients (31.6\%) had never been evaluated for COPD before the index hospital admission.

Mean Body Mass Index (BMI) was 26.9 ( \pm 5.6$)$. Respiratory function data was available for $556 \mathrm{pa}-$ tients (59.7\%) and are reported in table 1. Following post-bronchodilator spirometry, COPD severity stages according to GOLD showed the following distribution: stage 0 at risk, 96 cases $(17.3 \%$ of cases with spirometry), stage I mild, 15 cases $(2.7 \%)$, stage II moderate 138 (24.8\%), stage III severe 230 $(41.7 \%)$, and stage IV very severe $75(13.5 \%)$.

In the 12 months before admission 717 subjects $(77.0 \%)$ had manifested at least one other episode of COPD exacerbation, the mean number of episodes being $2.43( \pm 1.48)$. Only $58.4 \%$ patients had received annual influenza vaccination, while patients receiving pneumococcal vaccine in the previous 5 years represented only $12.6 \%$.
In the 3 months before admission 539 patients $(57.9 \%)$ had been hospitalised at least once for another episode of exacerbation, the mean number of episodes being 1.62 ( \pm 1.16$) ; 144$ patients $(15.5 \%)$ had been hospitalised in the 3 months prior for other causes than COPD (mean number of episodes, $1.15 \pm 0.52)$.

In 404 patients (43.4\%) COPD was complicated by acute on chronic or acute respiratory failure. Before admission 310 patients $(33.3 \%)$ were already on long-term oxygen therapy. The number of uncomplicated COPD patients with at least one concomitant extra-pulmonary disorder was 269 (29\%). The mean number of co-morbidities was 2.11 (range 1-10), the distribution of the Charlson Index being 1 in $35.7 \%, 2$ in $20.1 \%$, and $\geq 3$ in $22.6 \%$ of cases $(21.7 \%$ not reported). Of 673 patients $(72.3 \%)$ with at least one co-morbidity 370 (54.9\%) had cardio-vascular disorders (heart failure or coronary or peripheral artery disease), 83 $(27.2 \%)$ had diabetes, $70(10.4 \%)$ cerebrovascular disorders, 43 (6.4\%) chronic renal failure, 43 $(6.4 \%)$ gastro-duodenal ulcer, $29(4.3 \%)$ liver disorders, other conditions being represented less frequently.

Clinical evaluation of patients revealed worsening cough in 697 (74.9\%), increasing volume of expectoration in $652(70.0 \%)$, purulence in 492 $(52.8 \%)$, and colour modification of sputum in 552 $(59.3 \%)$. Only $97(10.3 \%)$ had manifested increased temperature $\geq 37.5{ }^{\circ} \mathrm{C}$ (average temperature $36.8 \pm 0.7{ }^{\circ} \mathrm{C}$ ). Mean respiratory frequency was $22.1 \pm 5.4$ breaths per minute, and mean heart rate was $92.4 \pm 16.1$ beats per minute. Mean blood

Table 1. - Respiratory function data, obtained in a stable phase (spirometry available for 556 patients, residual volume for 336, and CO transfer for 145 patients) and blood gas values at admission (896 patients, $96.2 \%)$. Data as mean \pm standard deviation (SD)

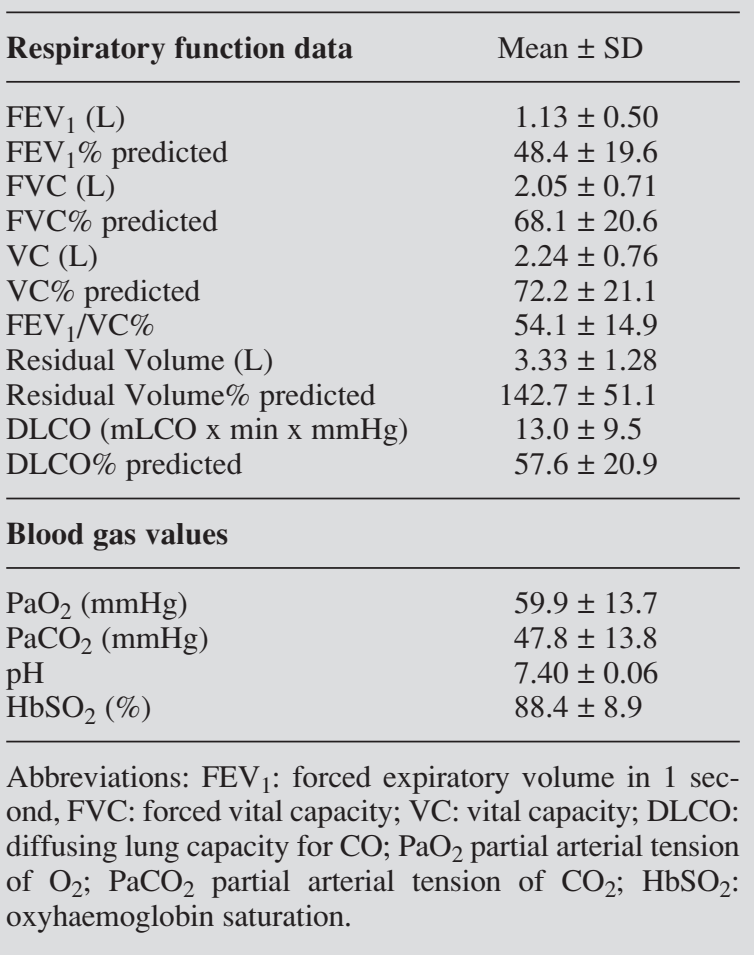


pressure was $140.3 \pm 20.6 / 81.7 \pm 11.2 \mathrm{mmHg}$ for systolic and diastolic pressure respectively. Critical signs, such as mental confusion, tremors, decompensated right heart failure, lethargy and use of accessory respiratory muscles, were found in 97 (10.4\%), 95 (10.2\%), 248 (26.3\%), 42 (4.5\%), and $256(27.5 \%)$ cases, respectively.

Blood gas values at admission are shown in table 1

At least one sample for microbiological study was obtained in 683 patients $(73.4 \%$ ) of which 279 sputum (40.8\%), 71 bronchial aspirates (10.4\%), 11 blood cultures $(1.6 \%)$, the remaining cases being not specified in records. Pathogens were isolated in no more than $55 \%$ of samples. The bacteria most frequently isolated in the sputum (only $32.7 \%$ of positive cases) were Ps. aeruginosa, $5.4 \%, H$. influenzae $3.2 \%$, Enterobacteriacee $2.9 \%$, S. aureus $2.5 \%, M$. catarrhalis $1.8 \%$. In the bronchial aspirates (54.8\% of positive cases) $H$. influenzae was most frequently isolated (16.9\%), followed by $H$. parainfluenzae (8.5\%), Ps. aeruginosa e S. pneumoniae (each 7.0\%), S. aureus and M. catarrhalis were $4.2 \%$ each. Serology and molecular biology were utilised in only 2.5 and $0.4 \%$ of cases, respectively.

At admission, usual drug therapy was modified in $96.8 \%$ of patients with a very scattered distribution of the different combinations of respiratory drugs, both topical and systemic. Among the 3 most represented combinations short acting $\beta_{2}$ agonists + corticosteroids + anticholinergic by inhalation + oral steroids was prescribed in $7.5 \%$ of patients, the same combination + theohylline in $5.1 \%$, and $\beta_{2}$ agonists + corticosteroids + anticholinergic by inhalation (no drugs by general route) in $3.8 \%$ of cases. Given that the large majority of patients $(89.3 \%)$ received an antibiotic treatment, if we consider the number of cases with a microbiological evaluation (see above) the drug would have been targeted in no more than one third of subjects. The main classes of antibiotics were cephalosporins $(24.1 \%$ of all treatments), fluoroquinolones $(22.4 \%)$, penicillin $+\beta$-lactamase inhibitor $(16.6 \%)$, and macrolides $(5.5 \%)$ as monotherapy; the most represented combinations were penicillins $+\beta$-lactamase inhibitor + fluoroquinolones $(5.4 \%)$, cephalosporins + fluoroquinolones $(4.9 \%)$, and cephalosporins + macrolides $(3.1 \%)$. Apart from bronchodilators, corticosteroids and antibiotics, 525 patients $(56.4 \%)$ received other drug or nonpharmacologic treatments, mainly represented by pulmonary rehabilitation (14.5\% of the global population), anti-smoking counselling $(8.1 \%$ of total co- hort but $51.0 \%$ of current smokers), oxygen therapy $(43.3 \%)$, non-invasive mechanical ventilation (12.7\%, mean duration 6.9 days, range $1-30)$, and invasive mechanical ventilation $(1.5 \%$, mean duration 7.75 days, range 2-28).

Admission to a respiratory intensive care unit was necessary in 36 patients (3.9\%) with a mean length of stay of $6.9 \pm 6.0$ days (range 1-28).

The percentage of patients receiving a definite respiratory treatment as prescribed in hospital is shown in figure 1.

Death occurred in 17 patients $(1.8 \%$ of the global case series, 9 patients for respiratory failure, 4 for cardiovascular failure and 2 for other causes, 12 had pre-existing chronic respiratory failure). Three patients died on invasive ventilation in ICU, 6 on non-invasive ventilation in ICU or ward.

The mean length of stay (LOS) was 12.0 \pm 7.1 (SD) days, range 1-75; the number of cases with a LOS $>12$ days was $302(32.4 \%)$.

An analysis of factors predicated on the main outcome measures (survival, length of stay, and need for mechanical ventilation) was conducted only for those 458 patients with a diagnosis of COPD confirmed by spirometry $\left(\mathrm{FEV}_{1} / \mathrm{VC} \leq 0.7\right.$ post-bronchodilator). The number of patients dying in hospital was too low to enable a reliable univariate logistic regression analysis on survival. The odds ratios of factors significantly predicting a LOS $>12$ days (mean LOS) or the need for mechanical ventilation are reported in table 2 .

During the 6-month follow up 355 patients (77.5\% of the cohort of patients with a correct diagnosis of COPD) were evaluated at the first month, $342(74.7 \%)$ at the third month and 322 $(70.3 \%)$ at the sixth month. The total number of patients who had died at 6 months was $19(5.3 \%$ of the follow up cohort).

A major outcome measure at follow up was rehospitalisation for a new episode of COPD exacerbation. A total number of 63 patients $(17.7 \%$ of all COPD subjects evaluable for such an outcome) had at least one episode of COPD exacerbation prompting a new hospitalisation. Bivariate logistic

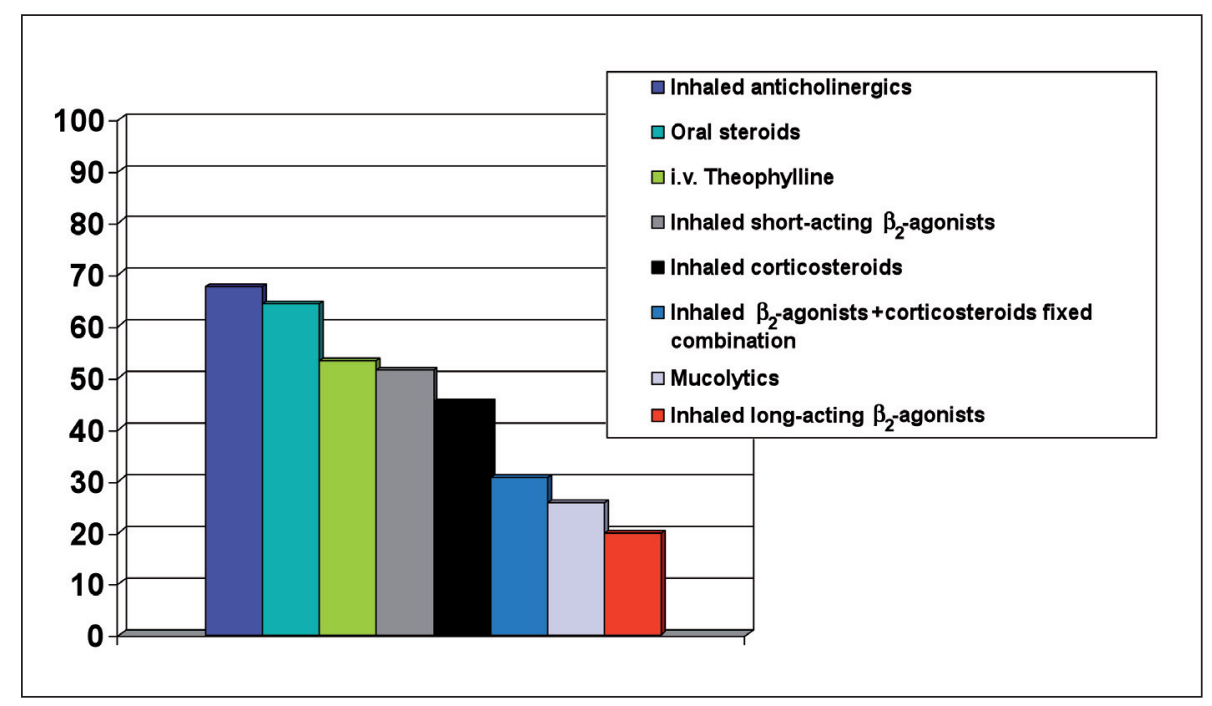

Fig. 1. - Percentage of patients receiving a definite respiratory treatment as prescribed in hospital (in decreasing order); for the main groups of non-fixed combination see "results" 
Table 2. - The odds ratios (OR) of factors significantly predicting a mean LOS >12 days or the need for mechanical ventilation

\begin{tabular}{lccc}
\hline Predict a $\mathbf{L O S}>\mathbf{1 2}$ days & P value & Odds Ratio & Confidence Interval 95\% \\
\hline Cigarette smoking (No vs. Yes) & 0.005 & 0.312 & $0.137-0.709$ \\
Haemoglobin (for each mg/dL of reduction) * & 0.02 & 1.239 & $1.032-1.487$ \\
$\mathrm{PaO}_{2} \leq 60$ mmHg (Yes vs. No) & 0.004 & 2.245 & $1.295-3.889$ \\
\hline Predict the need for mechanical ventilation & & & \\
\hline $\mathrm{pH} \leq 7.35$ (Yes vs. No) & $<0.001$ & 21.337 & $6.035-75.445$ \\
$\mathrm{Mental}$ confusion (Yes vs. No) & 0.01 & 9.908 & $1.689-58.143$ \\
$\mathrm{PaO} \leq 60$ mmHg (Yes vs. No) & 0.005 & 13.405 & $2.219-80.963$ \\
$\mathrm{BMI}$ * (for each unit increase above normal) & 0.035 & 1.123 & $1.008-1.251$ \\
Red blood cells * (n x 109/dL, for each " $\mathrm{n}$ " unit decrease) & 0.005 & 2.977 & $1.381-6.419$ \\
\hline * Continuous variable. & & &
\end{tabular}

regression analysis identified the following factors significantly increasing the risk of re-hospitalisation within 6 months: lower $\mathrm{FEV}_{1} \%$ predicted (OR $1.019, p=0.01 \mathrm{CI}: 1.005-1.035)$, and previous hospitalisations for a COPD exacerbation in the 12 months before (OR 2.73, $p=0.001 \mathrm{CI}$ : 1.49-4.9).

A multivariate analysis was conducted using the main significant variables as reported above. The predictors at 6 months that reached a statistically significant level for the outcome "new episode of COPD exacerbation" were the Charlson index (1 vs $>1$ ) [OR 1.71, $p=0.05 \mathrm{CI}: 1.01-2.91$ ], hospitalisation for a COPD exacerbation in the previous 12 months [OR 2.84, $p=0.0005$ CI: $1.59-5.1$ ], age $(<60$ vs $>80$ years) [OR 0.23, $p=0.017 \mathrm{CI}$ : 0.07-0.77], and COPD severity according to GOLD (very severe vs moderate) [OR 3.31, $p=0.006 \mathrm{CI}: 1.41-7.81$ ].

In the case of the outcome mortality the only significant predictor was the co-morbidity Charlson index (OR 10.3, $p=0.03 \mathrm{CI}$ : 1.25-84.96). The outcome hospitalisation was significantly predicted by hospitalisations for a COPD exacerbation in the previous 12 months (OR 3.59, $p=0.003 \mathrm{CI}$ : 1.54-8.39).

\section{Discussion}

The SOS study analysed a large cohort of COPD patients during and for 6 months after an episode of hospitalisation for an exacerbation, to evaluate actual clinical practice versus standards of care derived from international guideline recommendations, and to identify factors associated with a number of major outcomes such as survival, hospitalisation, length of stay, and further episodes of exacerbation.

The mean age of our population is similar to that reported in most other papers in the literature, a more important difference being the level of $\mathrm{FEV}_{1}$ about $10 \%$ predicted better than that reported in the majority of papers [1-8]. One possible explanation is in the recommendation given to investigators to register as baseline respiratory function data obtained in a stable phase in the 12 months before or 6 months after admission, since patients are often unable to perform a reliable spirometry during an exacerbation.
Quite surprisingly, only $60 \%$ of patients had a diagnosis correctly based on spirometry, the figure being similar to that of audit data reported in the literature [20-23]. Therefore according to current practice in hospital, at least in the case of Italy and the UK, about $40 \%$ of cases of COPD are defined only on a clinical basis.

The presence of definite risk factors, i.e. exposure to smoking or occupational/environmental pollutants, was absent in about $14 \%$ of the population. Data in the literature report a percentage of lifetime non-smokers with COPD from 5.5 [24] to $20 \%[25,26]$ but in many cases with other known risk factors. A risk of COPD overdiagnosis in never-smoker elderly subjects must not be overlooked [27]. Rather disturbing is the fact that about $1 / 3$ of patients were diagnosed with COPD for the first time only at the index hospitalisation, i.e. diagnosis was obtained very late in the natural course of the disease.

The distribution of severity stages according to GOLD showed that about 1 in 4 cases was of moderate degree, and $55 \%$ were severe or very severe. A quota of $20 \%$ at stage 0 /stage 1 GOLD is very surprising and poses a question of correct differential diagnosis between COPD and asthma or restrictive disorders.

The mean number of exacerbation episodes was comparable to average data in the literature (2.4 episodes per year) [28].

The main clinical factors characterising an exacerbation were dyspnea and worsening cough and expectoration volume (in about $3 / 4$ of the population), quite in agreement with the definition of an exacerbation as reported by GOLD guidelines [16].

\section{Standards of care}

In $73 \%$ of cases sampling for microbiology investigation was obtained but the reduced rate of positive results (54\% or less according to the type of sampling) and the well known problem of airways chronic colonisation stress the unresolved question of the real usefulness of microbiology in the clinical management of COPD exacerbations [29]. In our study about $1 / 3$ of patients could have 
received a targeted antibiotic according to an antibiogram, but this is a theoretical figure since many treatments are started empirically and are not changed if not unsuccessful. From an epidemiological point of view it is interesting to note the higher prevalence of Gram-negative bacteria, as expected from the average severity degree [29]. Real standards of care are difficult to define in this area, anyway this is a point in favour of obtaining nevertheless microbiological data; if seldom useful in the individual case, it can still be important for guiding local epidemiology-based empirical treatments.

In relation to drug treatments during the hospital stay it is very difficult to identify definite criteria given the large distribution of different combinations. It seems that the most frequent behaviour was to potentiate bronchodilator treatment and administer corticosteroids by oral or parenteral route in agreement with GOLD guidelines [16]. Among bronchodilators, i.v. theophylline represented a very frequent option (53.1\% of cases) despite the fact that GOLD guidelines consider methylxanthines as second line for the low therapeutic index [16]. The main classes of antibiotics used were fluoroquinolones and cephalosporins underlining a difference with respect to European guidelines where penicillin or penicillin $+\beta$-lactamase inhibitor are recommended as the first choice and fluoroquinolones as the second, apart from cases at risk for $P$. aeruginosa [30].

It is very important to underline in this section that several aspects of the diagnostic or treatment practice appear far distant from what is recommended by international guidelines: spirometry available only in $59.7 \%$ of cases, pulmonary rehabilitation prescribed to only $14.5 \%$ of subjects, anti-smoking counselling to only $8.1 \%$ (but representing $50 \%$ of current smokers), influenza and pneumococcal vaccination to only $58 \%$ and $13 \%$ respectively. Also performance indicators, in particular the 6-min walking test, and dyspnea scales were underutilised, with the result that we could calculate a composite score such as the BODE only in a minority of patients (about 10\%) and so were prevented from testing this as a prognostic factor in our population.

It is curious to note that all patients underwent a chest X-ray (necessary to exclude pneumonia), the large majority were assessed for arterial blood gases, but many had not undergone or were not asked to undergo spirometry to confirm COPD at least after the exacerbation episode. Similar data was reported by Roberts [20] for spirometry in relation to UK hospitals, but a better attitude emerged in Italian compared to UK hospitals versus smoking cessation (50\% versus $30 \%)$, although it was lower than desired. A positive point was the total coverage of patients with chronic respiratory failure with long-term oxygen therapy (or non-invasive mechanical ventilation in a minority of patients with ventilatory failure).

\section{Outcomes}

In-hospital survival compares favourably with respect to data obtained from other countries, in- hospital mortality being about 4 times lower $[2,6$, $8,22,23]$. Conversely, a very large, unselected, case series from the USA showed a similar mortality during hospitalisation, far lower than other studies carried out in the USA [31]. The differences are probably due to admission criteria, i.e. a large unselected population, such as in Patil's and our studies, versus case series of more severe patients selected according to a definite study design, or according to the type of hospital performing the study (e.g. tertiary referral centers are more likely to admit more severe patients).

The length of stay was on average longer in Italian hospitals (mean 12, median 9 days) than in the UK (mean 8.7, median 6 days) [22] or USA (median 9 days) [2]. Price et al. [22] demonstrated a relationship between organisational scores and LOS. In our study the contribution of organisational aspects or treatments to LOS was not assessed. Whether there is a relationship between longer LOS and better survival would probably merit further investigation.

The identification of factors influencing the major outcomes during the index hospitalisation underlined the importance of co-morbidities, hypoxaemia, respiratory acidosis, anemia, mental confusion, and tobacco smoking.

At follow up survival of COPD patients compared favourably with respect to other data in the literature showing a death rate at 6 months 2 to 4 times higher $[2,5,8]$, the most probable explanation being the different inclusion criteria and baseline severity of patients. Survival at follow up was mainly influenced by the Charlson index, i.e. comorbidities. The risk of a new exacerbation was significantly associated with previous hospitalisations, advanced age, disease severity and co-morbidities.

In COPD patients requiring hospitalisation for a new exacerbation, risk factors for re-hospitalisation were mainly low $\mathrm{FEV}_{1}$, and previous hospitalisation(s) for a COPD exacerbation in the previous year.

All the above risk factors have been variously reported in the literature [1-8], but according to our data the most reproducible and consistent risk factor seems to be previous hospital admission for a COPD exacerbation, in agreement with a recent study from Soler-Cataluña JJ et al. [7]. As a consequence, for a correct allocation of health care resources patients discharged from hospital after a COPD exacerbation should probably be admitted to home care or outpatient programmes with active surveillance, according to recent recommendations [18].

Are the risk factors underlined by our study amenable to prevention or early intervention? In our study, interventions known to have a preventive action, such as vaccinations, were underprescribed. Also in the case of rehabilitation the number of patients enrolled in a programme of pulmonary rehabilitation was very low, despite scientific evidence of their efficacy in reducing the risk of re-hospitalisation for COPD [32].

The strengths of our study were the prospective collection of data, and the large cohort of uns- 
elected patients enrolled at multiple hospital centers. It can be presumed that non-selected cohorts are more representative of a population of COPD patients [31] and probably more useful for the correct allocation of health care resources at a general level.

Among the weaknesses of our study we should mention the short follow up (6 months, mainly due to the resources available) and a significant number of subjects with questionable COPD diagnosis according to spirometry performed in a stable phase.

In conclusion, an important gap was found between current standards of care and recommendations, in particular in the use of spirometry for diagnosis and severity stratification along with a sub-optimal use of several management options. Hospitalisation per se was confirmed as a major determinant of further exacerbations and hospitalisations since COPD patients with a second hospitalisation within 12 months for an exacerbation had about 3 times the risk of a new exacerbation and new hospitalisation in the following 6 months.

Acknowledgments. The Italian SOS COPD study is a project of AIMAR (Associazione Scientifica Interdisciplinare per lo Studio delle Malattie Respiratorie), a scientific association for the multidisciplinary study of respiratory disorders. The study was supported by an unrestricted grant by Pfizer-Italy, Rome, and Boehringer-Ingelheim Milan, Italy.

We thank the following Italian Pulmonary Medicine Specialists and Departments that participated in the study: Alimonti Pietro, Divisione di Medicina e Pneumologia, Ospedale San Pietro, Roma; Balduin Renato, Unità Operativa Complessa di Pneumologia, U.S.S.L. 13 - Presidio Ospedaliero di Dolo (VE); Balestriero Claudio, Unità Operativa di Pneumologia, Ospedale Mater Salutis, Legnago (VR); Barbato Natalino, Divisione di Pneumologia, Presidio Ospedaliero G. Da Procida, Salerno; Barbera Santi, II Divisione Pneumologia-Pneumologia Oncologica, Presidio Ospedaliero Mariano Santo, Cosenza; Bosio Giancarlo, Unità Operativa di Pneumologia, Azienda Istituti Ospedalieri di Cremona; Bruna Severino, Divisione di Pneumotisiologia, Ospedale San Luigi, Orbassano (TO); Brunetta Franco, Unità Operativa di Pneumologia, Ospedale di Circolo, Varese; Capaccio Damiano, Unità Operativa di Endoscopia e Fisiopatologia Respiratoria, Presidio Ospedaliero Maria SS. Addolorata, Eboli (SA); Caputi Mario, Unità Operativa Complessa Malattie, Fisiopatologia e Riabilitazione Respiratoria, Azienda Ospedaliera Monaldi, Caserta; Carlone Stefano, Divisione Malattie Apparato Respiratorio, Azienda Ospedaliera San Giovanni dell'Addolorata, Roma; Casali Lucio, Struttura Complessa di Malattie dell'Apparato Respiratorio, Università degli Studi di Perugia; Casalini A. Gianni, Unità Operativa di Pneumologia ed Endoscopia Toracica, Azienda Ospedaliera, Parma; Cavalli Alberto, Divisione di Pneumologia, Policlinico "Sant'Orsola Malpighi”, Bologna; Cerri Ettore, Pneumotisiologia - Pad. Patologie Complesse, Ospedale San Martino, Genova; Ciccarelli Michele, Divisione di Pneumologia, Istituto Clinico Humanitas, Rozzano (MI); Cioffi Riccardo, Divisione di Malattie dell'Apparato Respiratorio, Azienda Ospedaliera San Sebastiano, Caserta; Confalonieri Marco, Struttura Complessa di Pneumologia, Azienda Ospedaliera "Ospedali Riuniti”, Trieste; Cottini Marcello, Unità Operativa di Pneumologia - Allergologia, Ospedali Riuniti, Bergamo; Cremona George, Divisione di Pneumologia, Ospedale San Raffaele, Milano; De Benedetto Fernando, Unità Operativa Complessa di Pneumologia, Presidio Ospedaliero Clinicizzato "SS. Annunziata", Chieti; De Francesca Francesco, Pneumotisiologia, Azienda Ospedaliera Pugliese
Ciaccio, Catanzaro; Del Donno Mario, Unità Operativa Complessa di Pneumologia, Azienda Ospedaliera G. Rummo, Benevento; Desideri Massimiliano, Unità Operativa di Pneumologia, Ospedale di Livorno; Donato Giovanni, Malattie Apparato Respiratorio, Azienda Ospedaliera Villa Sofia - CTO, Palermo; Failla Giuseppe, Divisione di Pneumologia, Azienda Ospedaliera Sant'Andrea, Roma; Faloppa Claudio, Medicina $1^{\circ}$ - Ospedale Pontedera, Azienda U.S.L. $\mathrm{N}^{\circ}$ 5, Pisa; Ferretti Gabriele, Struttura Operativa Complessa di Pneumologia, Azienda Ospedaliera SS. Antonio e Biagio, Alessandria; Fiore Donati Alfeo, Malattie Apparato Respiratorio, Ospedale San Salvatore, L'Aquila; Franceschi Paolo, Struttura Semplice di Pneumologia-Struttura Complessa Medicina Interna 1, Ospedale San Paolo, Savona; Gioia Vito, Unità Operativa di Pneumologia, Presidio Ospedaliero Ingrassia, Palermo; Giorgio Vincenza, Unità Operativa Complessa Malattie dell'Apparato Respiratorio e Riabilitazione Respiratoria, A.S.L. BA/4 - Polo Ospedaliero Bari Sud Ospedale F. Fallacara, Triggiano (BA); Giorgis Gian Enrico, Divisione di Pneumologia, Ospedale San Giovanni Bosco A.S.L. 4, Torino; Girbino Giuseppe, Dipartimento Clinico Malattie Respiratorie, Policlinico Universitario, Messina; Grillo Maria Patrizia, Dispensario d'Igiene - Ambulatorio Pneumologia, Presidio Ospedaliero di Tolmezzo (UD); Guffanti Enrico, Unità Operativa Pneumologia Riabilitativa, I.N.R.C.A. - IRCCS, Casatenovo (LC); Iannaccone Antonio, Divisione di Pneumologia, Ospedale San Giuseppe Moscati, Avellino; Isidori Pierpaolo, Unità Operativa di Pneumologia, Ospedale Santa Croce, Fano (PU); Leggieri Ennio, Medicina Seconda, Istituto Policlinico San Donato, San Donato Milanese (MI); Lo Coco Albino, Pneumologia, Ospedale Civico di Palermo, Palermo; Maspero Anna, Divisione di Pneumologia, Azienda Ospedaliera Sant'Anna, Como; Melani Andrea, Unità Operativa Complessa Fisiopatologia e Riabilitazione Respiratoria, Azienda Ospedaliera Universitaria Senese, Siena; Meregalli Guglielmo, Pneumologia, Ospedale San Gerardo, Monza; Milani Gianfranco, Struttura Operativa Complessa di Pneumologia, U.L.S.S. 18 - Ospedale Santa Maria della Misericordia, Rovigo; Montagner Paola, Reparto di Medicina Maschile, Azienda C. Poma; Mantova- Ospedale di Pieve di Coriano (MN); Naldi Mario, Unità Operativa di Broncopneumologia, Ospedale Civile San Donato U.S.S.L. 8, Arezzo; Nardini Stefano, Unità Operativa Pneumo -Tisiologia, Ospedale di Vittorio Veneto (TV); Negrin Rolando, Unità Operativa di Pneumologia, U.S.S.L. 6 Vicenza - Ospedale San Bortolo, Vicenza; Paggi Piero, Centro Broncopneumopatie, I.N.R.C.A. Ospedale Geriatrico, Ancona; Palamidese Alberto, Divisione di Pneumologia, Azienda Ospedaliera, Padova; Papalia Maria Antonella, Riabilitazione Pneumologica, Azienda Ospedaliera, Sondalo (SO); Pela Riccardo, Unità Operativa Complessa di Pneumologia, Ospedale Mazzoni, Ascoli Piceno; Pennisi Alfio, Malattie dell'Apparato Respiratorio, Casa di Cura Musumeci-Gecas, Catania; Penza Oronzo, Struttura Complessa di Pneumologia e U.T.I.R., Ospedale R. Silvestrini, Perugia; Peralta Giuseppe, Divisione di Pneumologia, Ospedale Cervello, Palermo; Politi Cecilia, Unità Operativa Complessa di Medicina Interna, Ospedale "F.Veneziale", Isernia; Rossi Aldo, Pneumologia, Azienda Ospedaliera "Ospedale San Salvatore", Pesaro; Sanguinetti Claudio, Unità Operativa Complessa di Pneumologia, Azienda Ospedaliera San Filippo Neri, Roma; Scaggiante Luciano, Unità Operativa di Pneumologia, Ospedale Civile Umberto I, Mestre (VE); Scatasta Mauro, Unità Operativa Medicina, Stabilimento Ospedaliero Boldrini, Tiene (VI); Schiavo Alfonso, Divisione di Medicina Interna, Presidio Ospedialero "Santa Maria Incoronata dell'Olmo", Cava dei Tirreni (SA); Scozzafava Mariano, Divisione di Pneumologia, Ospedale Maggiore, Lodi; Spagnolatti Liliana, Riabilitazione Cardio-Pneumologica, Ospedale San Sebastiano, Correggio (RE); Subiaco Silvano, Reparto di Pneumologia, Ospedale Murri, Jesi (AN); Sugamiele Mario, Malattie Apparato Respiratorio, Azienda Ospedaliera Sant'Antonio Abate, Erice (TP); Tondini Maurizio, Divisione di Pneumologia, Ospedale di Valle Camonica, Esine (BS); Tubaldi Alberto, Divisione di Pneumologia, Ospedale Civile, Macerata; Vincenzi Umberto, Struttura Complessa Malattie Apparato Respiratorio III, Azienda Ospedaliero-Universitaria "Ospedali Riuniti”, Foggia. 


\section{References}

1. Seneff MG, Wagner DP, Wagner RP, Zimmerman JE, Knaus WA. Hospital and 1-year survival of patients admitted to intensive care units with acute exacerbation of chronic obstructive pulmonary disease. JAMA 1995; 274: 1852-1857.

2. Connors AF, Dawson NV, Thomas C, et al. Outcomes following acute exacerbation of severe chronic obstructive pulmonary disease. Am J Respir Crit Care Med 1996; 154: 959-967.

3. Garcia-Aymerich J, Barreiro E, Farrero E, Marrades RM, Morera J, Anto JM, and the EFRAM investigators. Patients hospitalized for COPD have a high prevalence of modifiable risk factors for exacerbation (EFRAM study). Eur Respir J 2000; 16: 1037-1042.

4. Garcia-Aymerich J, Monsò E, Marrades RM, Escarrabill J, Fèlez MA, Sunyer J, Antò JM, and the EFRAM Investigators. Risk Factors for Hospitalisation for a Chronic Obstructive Pulmonary Disease Exacerbation. EFRAM STUDY. Am J Respir Crit Care Med 2001; 164: 1002-1007.

5. Almagro P, Calbo E, Ochoa de Echaguen A, et al. Mortality after hospitalisation for COPD. Chest 2002; 121: 1441-1448.

6. Groenewegen KH, Schols AMWJ, Wouters EFM. Mortality and mortality-related factors after hospitalisation for acute exacerbation of COPD. Chest 2003; 124: 459-467.

7. Soler-Cataluña JJ, Martínez-García MA, Román Sánchez P, Salcedo E, Navarro M, Ochando R. Severe acute exacerbations and mortality in patients with chronic obstructive pulmonary disease. Thorax 2005; 60: 925-931.

8. Gunen H, Hacievliyagil SS, Kosar F, et al. Factors affecting survival of hospitalised patients with COPD. Eur Respir J 2005; 26: 234-241.

9. Miravitlles M, Murio C, Guerriero T, Gisbert R, for the DAFNE Study Group. Pharmacoeconomic evaluation of acute exacerbations of chronic bronchitis and COPD. Chest 2002; 121: 1449-1455.

10. Mannino DM. COPD: epidemiology, prevalence, morbidity, and mortality, and disease heterogeneity. Chest 2002; 121: 121S-126S.

11. Lucioni C, Donner CF, De Benedetto F, et al. I costi della broncopneumopatia cronica ostruttiva in Italia: la fase prospettica dello studio ICE. Pharmacoeconomics Italian Research Articles 2005; 7: 119-134.

12. Celli BR, Cote CG, Marin JM, Casanova C, et al. The body-mass index, airflow obstruction, dyspnea, and exercise capacity index in chronic obstructive pulmonary disease. N Engl J Med 2004; 350: 1005-1012.

13. Ong K-H, Earnest A, Lu S-J. A multidimensional grading system (BODE Index) as predictor of hospitalisation for COPD. Chest 2005; 128: 3810-3816.

14. Roberts CM, Barnes S, Lowe D, Pearson MG, on behalf of the Clinical Effectiveness Evaluation Unit, Royal College of Physicians and the Audit Subcommittee of the British Thoracic Society. Evidence of a link between mortality in acute COPD and hospital type and resources. Thorax 2003; 58: 947-949.

15. Price LC, Lowe D, Hosker H, Anstey K, Pearson M, Roberts CM. The UK National COPD Audit 2003: impact of hospital resources and organisation of care on patient outcome following admissions for acute COPD exacerbation. Thorax 2006; 61: 837-842.

16. Global Strategy for the Diagnosis, Management and Prevention of COPD, Global Initiative for Chronic Obstructive Lung Disease (GOLD) 2006. Available from: http://www.goldcopd.org.

17. Barr RG, Bourbeau J, Camargo CA, Ram FSF. Tiotropium for stable chronic obstructive pulmonary disease: a meta-analysis. Thorax 2006; 61: 854-862.
18. Casas A, Troosters T, Garcia-Aymerich J, Roca J, Hernandez C, Alonso A, del Pozo F, de Toledo P, Antò JM, Rodriguez-Roisin R, Decramer M and members of the CHRONIC project. Integrated care prevents Hospitalizations for exacerbations in COPD patients. Eur Respir $J$ 2006; 28: 123-130.

19. Charlson ME, Pompei P, Ales KL, McKenzie CR. A new method of classifying prognostic comorbidity in longitudinal studies: development and validation. $J$ Chronic Dis 1987; 40: 373-383.

20. Roberts CM, Ryland I, Lowe D, Kelly Y, Bucknall CE, Pearson MG, on behalf of the audit sub-committee of the standards of care committee of the British Thoracic Society and the Clinical Effectiveness and Evaluation unit at the Royal College of Physicians. Audit of acute admissions of COPD: standards of care and management in the hospital setting. Eur Respir J 2001; 17: 343-349.

21. Roberts CM, Lowe D, Bucknall CE, Ryland I, Kelly Y, Pearson MG, on behalf of the British Thoracic Society Audit sub-committee of the standards of care committee and the Royal College of Physicians of London. Clinical audit indicators of outcome following admission to hospital with acute exacerbation of chronic obstructive pulmonary disease. Thorax 2002; 57: 137-141.

22. Price LC, Lowe D, Hosker HSR, Anstey K, Pearson MG, Roberts CM, on behalf of the British Thoracic Society and the Royal College of Physicians Clinical Effectiveness Evaluation Unit (CEEu). UK National COPD Audit 2003: impact of hospital resources and organisation of care on patient outcome following admission for acute COPD exacerbation. Thorax 2006; 61: 837-842.

23. Connolly MJ, Lowe D, Anstey K, Hosker HSR, Pearson MG, Roberts CM and on behalf of the British Thoracic Society and the Royal College of Physicians Clinical Effectiveness Evaluation Unit (CEEu). Admissions to hospital with exacerbations of chronic obstructive pulmonary disease: effect of age related factors and service organisation. Thorax 2006; 61: 843-848.

24. Kornmann O, Beeh KM, Beier J, Geis UP, Ksoll M, Buhl R. Newly diagnosed chronic obstructive pulmonary disease. Clinical features and distribution of the novel stages of the Global Initiative for Obstructive Lung Disease. Respiration 2003; 70: 67-75.

25. Viegi G, Scognamiglio A, Baldacci S, Pistelli F, Carrozzi L. Epidemiology of chronic obstructive pulmonary disease (COPD). Respiration 2001; 68: 4-19.

26. Anto JM, Vermeire P, Vestbo J, Sunyer J. Epidemiology of chronic obstructive pulmonary disease. Eur Respir J 2001; 17: 982-994.

27. Hardie JA, Buist AS, Vollmer WM, Ellingsen I, Bakke PS, Mørkve O. Risk of over-diagnosis of COPD in asymptomatic elderly never-smokers. Eur Respir $J$ 2002; 20: 1117-22.

28. Sethi S, File TM. Managing patients with recurrent acute exacerbations of chronic bronchitis: a common clinical problem. Curr Med Res Opin 2004; 20: 15111521.

29. Lode H, Allewelt M, Balk S, et al. A Prediction Model for Bacterial Etiology in Acute Exacerbations of COPD. Infection 2007; 35: 143-149.

30. Woodhead M, Blasi F, Ewig S, et al. Guidelines for the management of adult lower respiratory tract infections. Eur Respir J 2005; 26: 1138-1180.

31. Patil SP, Krishnan JA, Lechtzin N, Diette GB. In-hospital mortality following acute exacerbations of COPD. Arch Intern Med 2003; 163: 1180-1186.

32. Garcia-Aymerich J, Farrero E, Felez MA, Izquierdo J, Marrades RM, Antó JM, Estudi del Factors de Risc d'Agudització de la MPOC investigators. Risk factors for readmision to hospital for a COPD exacerbation: a prospective study. Thorax 2003; 58: 100-105. 